TAPROBANICA, ISSN 1800-427X. June, 2014. Vol. 06, No. 01: pp. 49-52.

(C) Taprobanica Private Limited, 146, Kendalanda, Homagama, Sri Lanka.

http://www.sljol.info/index.php/tapro

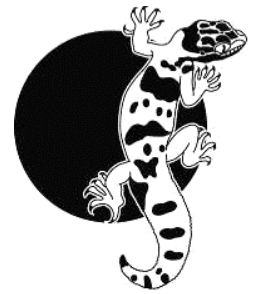

\section{Marine Mollusc Diversity along the Southwest Coast of Sri Lanka}

Molluscan species as well as class-level diversity is highest in the marine environment (Russell-Hunter, 1983). The current survey data reveals that Sri Lanka is inhabited by about 240 species of marine molluscs belonging to four of the seven classes representing marine molluscs (De Silva, 2006). The study area, along the southwest coast of Sri Lanka, experiences the southwest monsoon from May to September, which has a significant impact on climate and oceanographic conditions in this region.

Sites were selected including those associated with rocky habitats. Such as, isolated rocks on sandy beaches or scattered continuous rocks along the shoreline. Shells were collected along a $100 \mathrm{~m}$ line transect parallel to the shoreline, along the backshore. Live specimens encountered along each transect were identified using field guides (Abbott, 1991; Allen, 1998; Dyerly et al., 1998; Eye, 1989; Kirthisinghe, 1978; Sabelli, 1979; Woodward, 1998), data were recorded and specimens were released. The field survey was carried-out during the periods of August 2008 to September 2008 and December 2008 to March 2009 along the shores of Chilaw, Negambo, Panadura and Tangalle (Fig. 1). Observations recorded include GPS coordinates, types of algae present, wave action and slope. GPS points were obtained using a hand-held GPS receiver. Algal samples were collected and identified using a field guide (Fish et al., 1989). The frequency of wave action was observed as the number of waves striking the breakwater per minute. Slope was measured using a clinometer (Brunton Survey Master 360 LA Sighting). The collected shells were cleaned, separated and identified using field guides above cited. The identification was further confirmed with identified shell collections at the national museum of Sri Lanka (NMSL). Analysis was preliminarily carried out using the Shannon-
Weiner diversity index. Further analysis was carried out using Cluster and Principle Component Analysis in order to investigate variation in habitat with regard to the distribution and abundance of the species recorded during the study.

The results for Shannon-Weiner index revealed a highest diversity at Tangalle (3.37) and Negambo (3.36), highest evenness in Negambo (0.051). Panadura showed the lowest diversity (2.68) and the lowest evenness (0.035) (Table 1). Some shells were found only at a single site. Several species including Amathina tricarinata, Asaphis deflorata, Cantharus undosus, Chicoreus brunneus, Conus figulinus, Dentallium sp., Ficus sp., Globularia fluctuata, Mesodesma glabratum, Phalium decussatum, Pharaonella sp., Terebra sp., Tonna luteostoma and Vasticardium assimile were only recorded during one season (Appendix 1). It was found that the conditions of the shore differs with the season, algal species were distinct by season (Appendix 2). As for example while there was just one species of alga found on an isolated rock at the Panadura site during the monsoon, after the monsoon we observed numerous species of algae as well as marine invertebrate species among the algae such as Chiton sp. Though more data are needed, there were several species commonly found at all sites studied (Appendix 1). Of the commonly found species, there were 12 bivalves and five gastropods belonging to 13 families, along with one unidentified species. Although these species were found at all sites, their abundance varied at the different sites (Fig. 1) and also with regard to the monsoon (Fig. 2-3). The sites were grouped into four clusters at the $25 \%$ phenon level and two groups at $100 \%$ phenon level (Figs. 4 A-D).

The overall results revealed 53 species of gastropods belonging to 29 families, 52 species of bivalves representing 16 families, and one species each representing classes Cephalopoda, 
Scaphopoda and Polyplacophora. Considering the commonly found species at all four sites, we propose that presence is correlated with different habitat types, except in some special cases such as Duplicaria raphanula and Perna virdis. In these cases other factors may also be at play in determining distributions, including predation pressure, harvest by humans for food or other economic interests, natural dispersal ability, presence of different food resources, near shore current movements, etc. In addition our data suggest that species abundance differs with the monsoonal conditions. Our survey results, while showing some interesting patterns, are preliminary and warrant further investigation.

Table 1: Shannon-Wiener indices; C, Chilaw; N, Negombo; P, Panadura; T, Tangalle; NE, northeastern monsoon; SW, southwestern monsoon.

\begin{tabular}{|l|c|c|c|c|}
\hline & $\mathbf{N}$ & $\mathbf{C}$ & $\mathbf{P}$ & $\mathbf{T}$ \\
\hline $\mathbf{H}$ & 3.36 & 2.83 & 2.68 & 3.37 \\
\hline $\mathbf{H}$ max & 4.19 & 4.06 & 4.33 & 4.32 \\
\hline $\mathbf{E}$ & 0.051 & 0.049 & 0.035 & 0.045 \\
\hline
\end{tabular}

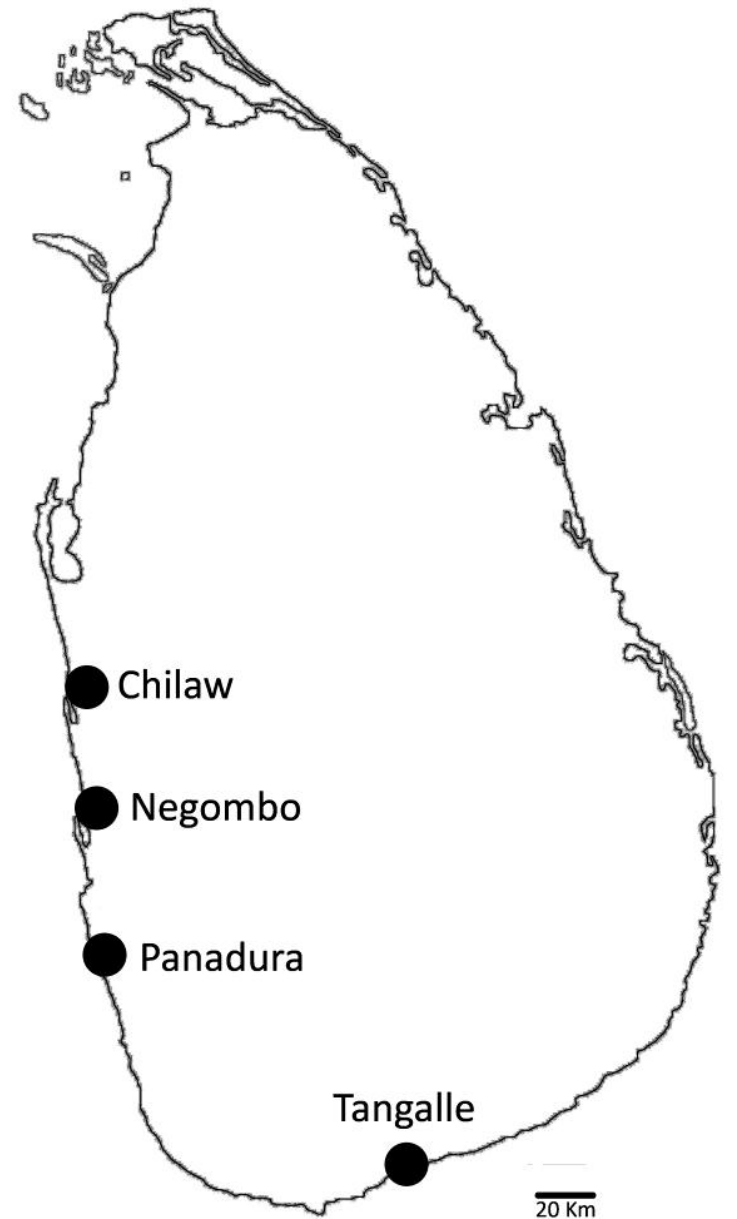

Figure 1: Study sites

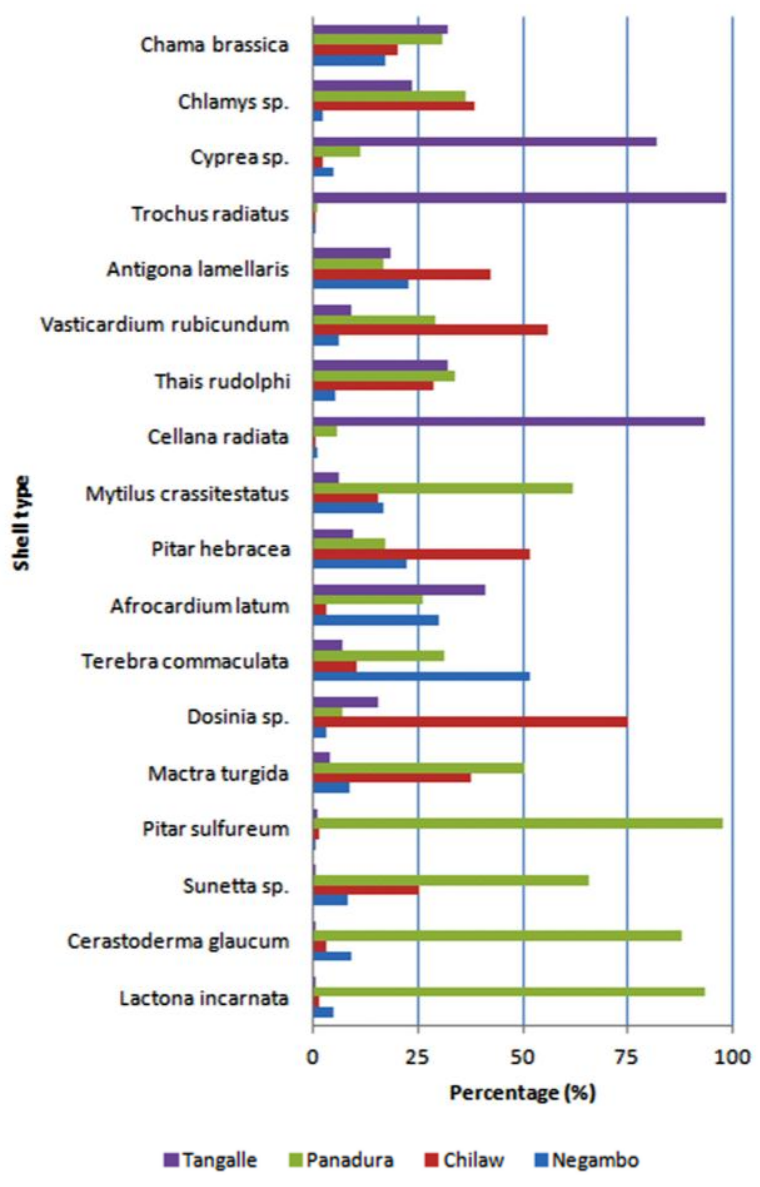

Figure 2: Relative abundance of selected species among commonly found shells at all four sites.

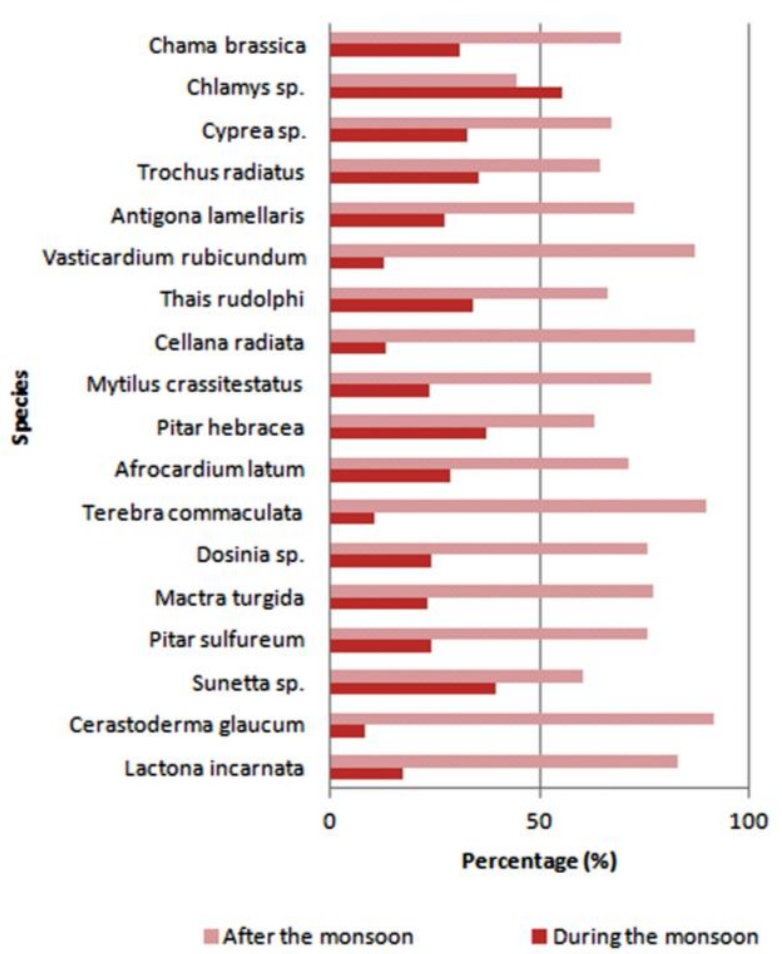

Figure 3: Relative abundance of selected species during and after the monsoon period. 


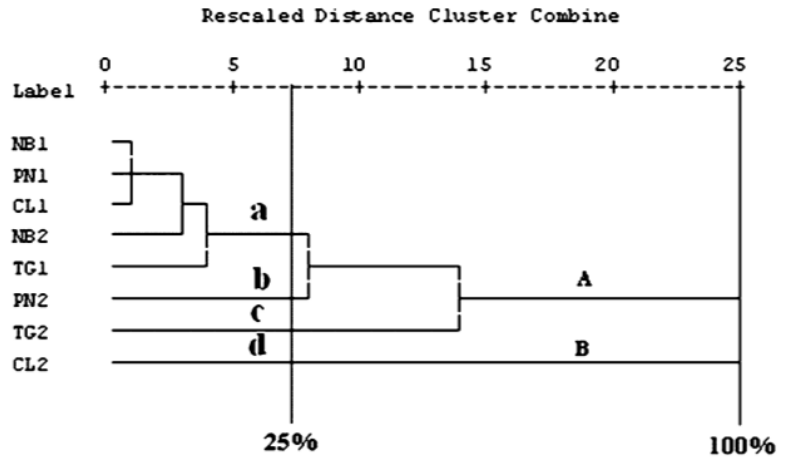

Figure 4: Dendrogram produced from the shell abundance data collected from different sites. Sites were grouped according to similarities of morphological shell characters; PN, Panadura; NB, Negombo; CL, Chilaw; TG, Tangalle; 1, visit 1; 2, visit 2.

\section{Literature cited}

Abbott, R. T., 1991. Sea shells of South east Asia, Tynron Press, Scotland: 145.

Allen, G. R., 1998. Marine life of Malaysia and the Indo-Pacific. In: Nicholes, F. and M. Stachels (eds.). Periplus editions [HK] Ltd, Singapore: 4249.

De Silva, D., 2006. Current status of taxonomy and ecology of marine molluscs Sri Lanka. The fauna of Sri Lanka: 274-287.

Dyerly, R., P. Fiene-Severns, and M. Severns, 1998. Tropical sea shells of India and Sri Lanka. In: Henderson, J. (ed.). Periplus editions [HK] Ltd, Singapore: 61.
Eye, K. R., 1989. Shells of the world. In: Bowling, S., M. Brown, R. Dawes, and R. Rees (eds.). Miette Street, London: 179.

Kirthisinghe, P., 1978. Sea shells of Sri Lanka. Tokyo. Charles E. Tuttle co. Inc.: 220.

Russel-Hunter, W. D., 1983. Overview: planetary distribution of and ecological constrains upon the mollusca. In: Wilber, K. M. and W. D. RusselHunter (Eds.). The mollusca. Academic press, Inc, Florida.

Sabelli, B., 1979. Guide to shells, Simon and Schuster Inc., New York: 512.

Woodward, F., 1998. Shells, The Apple press, London: 62.

Yoganathan, S., 2005. Monsoonal variations in the distribution of macro invertebrates of the intertidal zone at three locations in Mannar island, BSc. Thesis, University of Jaffna: 51.

Submitted: 23 Nov. 2013, Accepted: 02 April 2014 Section Editor: Brenden Holland

\section{H. T. Rubesinghe ${ }^{1} \&$ S. R. Krishnarajah ${ }^{2}$}

1 Centre for Environmental Justice, Kuruppu Road, Colombo 08, Sri Lanka E-mail: dentallium@gmail.com 2 Department of Zoology, Open University of Sri Lanka, Nawala, Nugegoda, Sri Lanka

Appendix 1: Species found at each site; C, Chilaw; N, Negombo; P, Panadura; T, Tangalle; NE, northeastern monsoon; SW, southwestern monsoon

\begin{tabular}{|c|c|c|c|c|c|c|c|c|c|}
\hline \multirow{2}{*}{ Species } & \multirow{2}{*}{$\begin{array}{l}\text { Season } \\
\text { Site } \\
\end{array}$} & \multicolumn{4}{|c|}{ SW } & \multicolumn{4}{|c|}{$\mathrm{NE}$} \\
\hline & & $\mathrm{N}$ & $\mathrm{C}$ & $\mathrm{P}$ & $\mathrm{T}$ & $\mathrm{N}$ & $\mathrm{C}$ & $\mathrm{P}$ & $\mathrm{T}$ \\
\hline \multicolumn{10}{|c|}{ Afrocardium latum } \\
\hline \multicolumn{10}{|c|}{ Amathina tricarinata } \\
\hline \multicolumn{10}{|c|}{ Anadara complanata } \\
\hline \multicolumn{10}{|c|}{ Antigona lamellaris } \\
\hline \multicolumn{10}{|c|}{ Arca bistrigata } \\
\hline \multicolumn{10}{|c|}{ Arca ventricosa } \\
\hline \multicolumn{10}{|c|}{ Architectonica sp. } \\
\hline \multicolumn{10}{|c|}{ Asaphis deflorata } \\
\hline \multicolumn{10}{|c|}{ Astralium sp. } \\
\hline \multicolumn{10}{|c|}{ Barbatia velata } \\
\hline \multicolumn{10}{|c|}{ Barbetia virescens } \\
\hline \multicolumn{10}{|c|}{ Bassina calophylla } \\
\hline \multicolumn{10}{|c|}{ Batillaria zonalis } \\
\hline \multicolumn{10}{|c|}{ Bulla amphula } \\
\hline \multicolumn{10}{|c|}{ Bursa awatii } \\
\hline \multicolumn{10}{|c|}{ Calista Chinensis } \\
\hline \multicolumn{10}{|c|}{ Callista erycina } \\
\hline Cantharus & sus & & & & & & & & \\
\hline
\end{tabular}

\begin{tabular}{|c|c|c|c|c|c|c|c|c|c|}
\hline \multirow{2}{*}{ Species } & \multirow{2}{*}{$\begin{array}{l}\text { Season } \\
\text { Site }\end{array}$} & \multicolumn{4}{|c|}{ SW } & \multicolumn{4}{|c|}{$\mathrm{NE}$} \\
\hline & & $\mathrm{N}$ & $\mathrm{C}$ & $\mathrm{P}$ & $\mathrm{T}$ & $\mathrm{N}$ & $\mathrm{C}$ & $\mathrm{P}$ & $\mathrm{T}$ \\
\hline \multicolumn{10}{|c|}{ Cardiata bicolor } \\
\hline \multicolumn{10}{|c|}{ Cardiata variegata } \\
\hline \multicolumn{10}{|c|}{ Cassostrea sp. } \\
\hline \multicolumn{10}{|c|}{ Cellana luchuna } \\
\hline \multicolumn{10}{|c|}{ Cellana radiata } \\
\hline \multicolumn{10}{|c|}{ Cerastoderma glaucum } \\
\hline \multicolumn{10}{|c|}{ Chama brassica } \\
\hline \multicolumn{10}{|c|}{ Chicoreus brunneus } \\
\hline \multicolumn{10}{|l|}{ Chiton Sp. } \\
\hline \multicolumn{10}{|c|}{ Chlamys sp. } \\
\hline \multicolumn{10}{|c|}{ Clypidina notata } \\
\hline \multicolumn{10}{|c|}{ Codakia punctata } \\
\hline \multicolumn{10}{|c|}{ Conus figulinus } \\
\hline \multicolumn{10}{|l|}{ Conus sp. } \\
\hline \multicolumn{10}{|c|}{ Crucibulum extinctorium } \\
\hline \multicolumn{10}{|c|}{ Cymatium retusum } \\
\hline \multicolumn{10}{|c|}{ Cymatium spengleri } \\
\hline Cyprea mo & & & & & & & & & \\
\hline
\end{tabular}




\begin{tabular}{|c|c|c|c|c|c|c|c|c|c|}
\hline \multirow{2}{*}{ Species } & \multicolumn{2}{|l|}{ Season } & \multicolumn{3}{|c|}{ SW } & \multicolumn{4}{|c|}{$\mathrm{NE}$} \\
\hline & Site & $\mathrm{N}$ & $\mathrm{C}$ & $\mathrm{P}$ & $\mathrm{T}$ & $\mathrm{N}$ & $\mathrm{C}$ & $\mathrm{P}$ & $\mathrm{T}$ \\
\hline \multicolumn{10}{|l|}{ Cyprea sp. } \\
\hline \multicolumn{10}{|c|}{ Dentallium sp. } \\
\hline \multicolumn{10}{|c|}{ Diodora mus } \\
\hline \multicolumn{10}{|c|}{ Donax incarnata } \\
\hline \multicolumn{10}{|c|}{ Donax scortum } \\
\hline \multicolumn{10}{|c|}{ Donax variabilis } \\
\hline \multicolumn{10}{|c|}{ Dosinia sp. } \\
\hline \multicolumn{10}{|c|}{ Drupa musiva } \\
\hline \multicolumn{10}{|c|}{ Drupa ricinus } \\
\hline \multicolumn{10}{|c|}{ Drupella concatenata } \\
\hline \multicolumn{10}{|c|}{ Duplicaria raphanula } \\
\hline \multicolumn{10}{|c|}{ Engina melanozona } \\
\hline \multicolumn{10}{|c|}{ Eplica versicolor } \\
\hline \multicolumn{10}{|c|}{ Euchelus atratus } \\
\hline \multicolumn{10}{|c|}{ Ficus sp. } \\
\hline \multicolumn{10}{|c|}{ Gastrana polygona } \\
\hline \multicolumn{10}{|c|}{ Globularia fluctuata } \\
\hline \multicolumn{10}{|c|}{ Gonilia calliglypta } \\
\hline \multicolumn{10}{|c|}{ Haliotis squamosa } \\
\hline \multicolumn{10}{|c|}{ Heliacus variegatus } \\
\hline \multicolumn{10}{|c|}{ Hipponyx pilosus } \\
\hline Irus irus & & & & & & & & & \\
\hline Latona fabo & & & & & & & & & \\
\hline Lima lima & & & & & & & & & \\
\hline Lioconchaf & giata & & & & & & & & \\
\hline Littorina $g r$ & lata & & & & & & & & \\
\hline Littorina un & & & & & & & & & \\
\hline Lunulicardi & bretusa & & & & & & & & \\
\hline Macroschis & & & & & & & & & \\
\hline Mactra turg & & & & & & & & & \\
\hline Mammillia & & & & & & & & & \\
\hline Megathura & iulata & & & & & & & & \\
\hline Meretrix me & & & & & & & & & \\
\hline Mesodesma & bratum & & & & & & & & \\
\hline Perna virid & & & & & & & & & \\
\hline Nassarius $p$ & dulus & & & & & & & & \\
\hline
\end{tabular}

\begin{tabular}{|c|c|c|c|c|c|c|c|c|c|}
\hline \multirow{2}{*}{ Species } & \multicolumn{2}{|l|}{ Season } & \multicolumn{3}{|c|}{ SW } & \multicolumn{4}{|c|}{$\mathrm{NE}$} \\
\hline & Site & $\mathrm{N}$ & $\mathrm{C}$ & $\mathrm{P}$ & $\mathrm{T}$ & $\mathrm{N}$ & $\mathrm{C}$ & $\mathrm{P}$ & $\mathrm{T}$ \\
\hline \multicolumn{10}{|l|}{ Natica sp. } \\
\hline \multicolumn{10}{|c|}{ Nerita plicata } \\
\hline \multicolumn{10}{|c|}{ Nerita textilis } \\
\hline \multicolumn{10}{|c|}{ Oliva sp. } \\
\hline \multicolumn{10}{|c|}{ Paphia malabarica } \\
\hline \multicolumn{10}{|c|}{ Periglypta reticulata } \\
\hline \multicolumn{10}{|c|}{ Petricola lapicida } \\
\hline \multicolumn{10}{|c|}{ Phalium decussatum } \\
\hline \multicolumn{10}{|c|}{ Pharaonella sp. } \\
\hline \multicolumn{10}{|c|}{ Pitar hebracea } \\
\hline \multicolumn{10}{|c|}{ Pitar sulfureum } \\
\hline \multicolumn{10}{|c|}{ Pyrene sp. } \\
\hline \multicolumn{10}{|c|}{ Rhinoclavis sinensis } \\
\hline \multicolumn{10}{|c|}{ Saccostrea sp. } \\
\hline \multicolumn{10}{|c|}{ Septifer bilocularis } \\
\hline \multicolumn{10}{|c|}{ Spirula spirula } \\
\hline \multicolumn{10}{|c|}{ Spondylus layardi } \\
\hline \multicolumn{10}{|c|}{ Spondylus Tenebrosus } \\
\hline \multicolumn{10}{|c|}{ Sunetta scripta } \\
\hline \multicolumn{10}{|l|}{ Sunetta sp. } \\
\hline \multicolumn{10}{|l|}{ Tellina sp. } \\
\hline \multicolumn{10}{|c|}{ Tellinides timorensis } \\
\hline Terebra sp. & & & & & & & & & \\
\hline Thais intern & & & & & & & & & \\
\hline Thais sp. & & & & & & & & & \\
\hline Tonna luteo & & & & & & & & & \\
\hline Trochus rac & & & & & & & & & \\
\hline Turbo sp. & & & & & & & & & \\
\hline Turritella ce & nunis & & & & & & & & \\
\hline Unidentifiec & & & & & & & & & \\
\hline Unidentified & & & & & & & & & \\
\hline Unknown l & & & & & & & & & \\
\hline Vasticardiu & ssimile & & & & & & & & \\
\hline Vasticardiu & ubicundum & & & & & & & & \\
\hline Zeuxis velat & & & & & & & & & \\
\hline
\end{tabular}

Appendix 2: Field data collected on the study sites; dm, during monsoon; af, after monsoon.

\begin{tabular}{|c|c|c|c|c|c|c|c|c|}
\hline Loc. & \multicolumn{2}{|c|}{ Chilaw } & \multicolumn{2}{|c|}{ Negambo } & \multicolumn{2}{|c|}{ Panadura } & \multicolumn{2}{|c|}{ Tangalle } \\
\hline GPS & \multicolumn{2}{|c|}{$7^{\circ} 34^{\prime} 22^{\prime \prime} \mathrm{N}, 79^{\circ} 48^{\prime} 8^{\prime \prime} \mathrm{E}$} & \multicolumn{2}{|c|}{$7^{\circ} 13^{\prime} 40^{\prime \prime} \mathrm{N}, 79^{\circ} 50^{\prime} 53^{\prime \prime} \mathrm{E}$} & \multicolumn{2}{|c|}{$6^{\circ} 42^{\prime} 53^{\prime \prime N}, 79^{\circ} 54^{\prime} 24^{\prime \prime E}$} & \multicolumn{2}{|c|}{$6^{\circ} 0^{\prime} 44^{\prime \prime} \mathrm{N}, 80^{\circ} 46^{\prime} 4^{\prime \prime} \mathrm{E}$} \\
\hline $\begin{array}{l}\text { Visit \& } \\
\text { date }\end{array}$ & $\begin{array}{c}\text { Visit 1 } \\
(\mathrm{dm}) \\
22.8 .2008\end{array}$ & $\begin{array}{c}\text { Visit } 2 \\
\text { (am) } \\
8.3 .2009\end{array}$ & $\begin{array}{c}\text { Visit } 1 \\
(\mathrm{dm}) \\
22.8 .2008\end{array}$ & $\begin{array}{c}\text { Visit } 2 \\
(\mathrm{am}) \\
8.3 .2009\end{array}$ & $\begin{array}{c}\text { Visit } 1 \\
(\mathrm{dm}) \\
16.8 .2008\end{array}$ & $\begin{array}{c}\text { Visit } 2 \\
(\mathrm{am}) \\
27.12 .2008\end{array}$ & $\begin{array}{c}\text { Visit } 1 \\
(\mathrm{dm}) \\
27.9 .2008\end{array}$ & $\begin{array}{c}\text { Visit } 2 \\
\quad(\mathrm{am}) \\
28.12 .2008\end{array}$ \\
\hline Slope $/^{0}$ & 5 & 7 & 8 & 5 & 6 & 20 & 11 & 7 \\
\hline $\begin{array}{l}\text { Wave } \\
\text { action/ } \\
\text { Swells } \\
\text { min }^{-1}\end{array}$ & 8 & 8 & 7 & 8 & 8 & 8 & 6 & 8 \\
\hline $\begin{array}{l}\text { Algae } \\
\text { genera }\end{array}$ & Ulva & $\begin{array}{c}\text { Gracilaria } \\
\text { Ulva }\end{array}$ & - & $\begin{array}{c}\text { Gracilaria } \\
\text { Ulva } \\
\text { Padina } \\
\text { Jania }\end{array}$ & Chaetomorpha & $\begin{array}{c}\text { Gracilaria } \\
\text { Ulva } \\
\text { Jania } \\
\text { Gelidium } \\
\text { Sargassum } \\
\end{array}$ & $\begin{array}{c}\text { Ulva } \\
\text { Sargassum } \\
\text { Caularpa } \\
\text { Halimida } \\
\text { Padina }\end{array}$ & $\begin{array}{c}\text { Ulva } \\
\text { Halimeda } \\
\text { Sargassum }\end{array}$ \\
\hline
\end{tabular}

